\title{
Modeling Red Blood Cell and Iron Dynamics in Patients with Chronic Kidney Disease
}

\author{
H. T. Banks ${ }^{1}$, Karen M. Bliss ${ }^{2}$ and Hien $\operatorname{Tran}^{1}$ \\ ${ }^{1}$ Center for Research in Scientific Computation \\ North Carolina State University \\ Raleigh, NC 27695 \\ ${ }^{2}$ United States Military Academy \\ West Point, NY 10996
}

February 10, 2012

\begin{abstract}
Chronic kidney disease causes a slow loss of kidney function over time and can eventually lead to End Stage Renal Disease, where a patient must undergo dialysis to remove fluids and wastes from the body. These patients also suffer from a lack of the hormone erythropoietin (EPO), produced in the kidneys, that stimulates red blood cell (RBC) production. Without intervention, patients suffer from anemia. Patients are treated with both EPO and iron in order to stimulate RBC production.

We develop a partial differential equation model for RBC dynamics using two structure variables, one for age and one for cellular iron endowment. We couple this with a set of ordinary differential equations modeling iron dynamics. We take into account the effects of both inflammation and neocytolysis, which are known to affect patients undergoing treatment.
\end{abstract}

Keywords: mathematical model, mathematical biology, erythropoiesis, erythrocyte, red blood cell, chronic kidney disease, dialysis, iron, neocytolysis, hepcidin, EPO, hemoglobin

e-mails: htbanks@ncsu.edu, kmbliss@ncsu.edu, tran@ncsu.edu 


\section{Introduction}

It is estimated that 31 million Americans have chronic kidney disease (CKD). Among those, approximately 330 thousand were classified as being in End-Stage Renal Disease (ESRD) and required dialysis [42] to remove wastes and fluids from the blood. For the purposes of this study, we consider only hemodialysis, where a patient's blood is exposed to a semipermeable membrane outside of the body.

In addition to regulating blood pressure and filtering waste products from blood, kidneys produce a hormone called erythropoietin (EPO) that is the major regulator of erythropoiesis, or red blood cell production. EPO level is normally controlled by a negative feedback mechanism in the kidneys, but patients in ESRD do not produce sufficient levels of EPO to maintain blood hemoglobin concentration. Hemoglobin is the protein that gives red blood cells the ability to carry oxygen. Patients with low hemoglobin concentration may present symptoms of anemia, such as decreased cardiac function, fatigue, and decreased cognitive function.

In order to prevent anemia, patients typically receive recombinant human EPO (rHuEPO) intravenously to stimulate red blood cell production. However, treatment is far from perfect. In 2006, only half of dialysis patients had a mean monthly hemoglobin greater than 11 grams per deciliter [42], the desired minimum level set by the National Kidney Foundation [29].

Iron is required to produce hemoglobin, and iron deficiency can be an issue among patients receiving $\mathrm{rHuEPO}$ therapy. Oral iron supplementation is often ineffective, so intravenous iron supplementation has become a mainstay in many patients undergoing rHuEPO therapy [25].

Iron availability is negatively affected by inflammation level in the body. Most patients with CKD have elevated levels of inflammation due to CKD and the presence of other medical issues (e.g., diabetes, hypertension, etc.) [26].

We develop a model for red blood cell populations using two structure variables, one for age-structuring and one to account for a cell's iron endowment. We couple these partial differential equations with a system of ordinary differential equations that models the iron cycle.

\section{Previous models}

The process of erythropoiesis has been modeled in several physiological scenarios. In [31], $\mathrm{rHuEPO}$ therapy is considered in healthy individuals. This model incorporates the negative feedback to endogenous EPO production. EPO is assumed to be cleared using MichaelisMenten dynamics. A similar model was used to fit data in rats [46]. Both of these models use delay instead of age-structured modeling.

Both [6] and [7] use age-structured models, as does the model described in [27], which assumes that the oldest mature erythrocytes will be destroyed, yielding a moving boundary condition. In [2], EPO is assumed to accelerate maturation of cells undergoing erythropoiesis. Additionally, EPO is assumed to be consumed during the process of erythropoiesis.

The model presented here is a significant departure from these models in that it incorporates iron. Additionally, we have accounted for the effects of inflammation and neocytolysis, a down-regulating mechanism by which red blood cells are selectively destroyed when blood 
oxygen level rises.

The authors have previously presented a more simplified version of this model in $[5,8]$, including simulations. The simplified model is significantly less sophisticated; assumptions were made there so that simulations would be more tractable.

\section{Overview of Red Blood Cell Production}

Erythrocytes are produced primarily from stem cells in bone marrow. In the presence of certain hormones, stem cells divide asymmetrically, producing a committed colony-formingunit (CFU) while maintaining the population of stem cells. Erythrocyte lineage continues as depicted in Figure 1: erythroid burst-forming unit (BFU-E), erythroid colony-formingunit (CFU-E), proerythrocyte, basophilic erythrocyte, polychromatic erythroblast, orthochromatic erythroblast, reticulocyte, and erythrocyte.

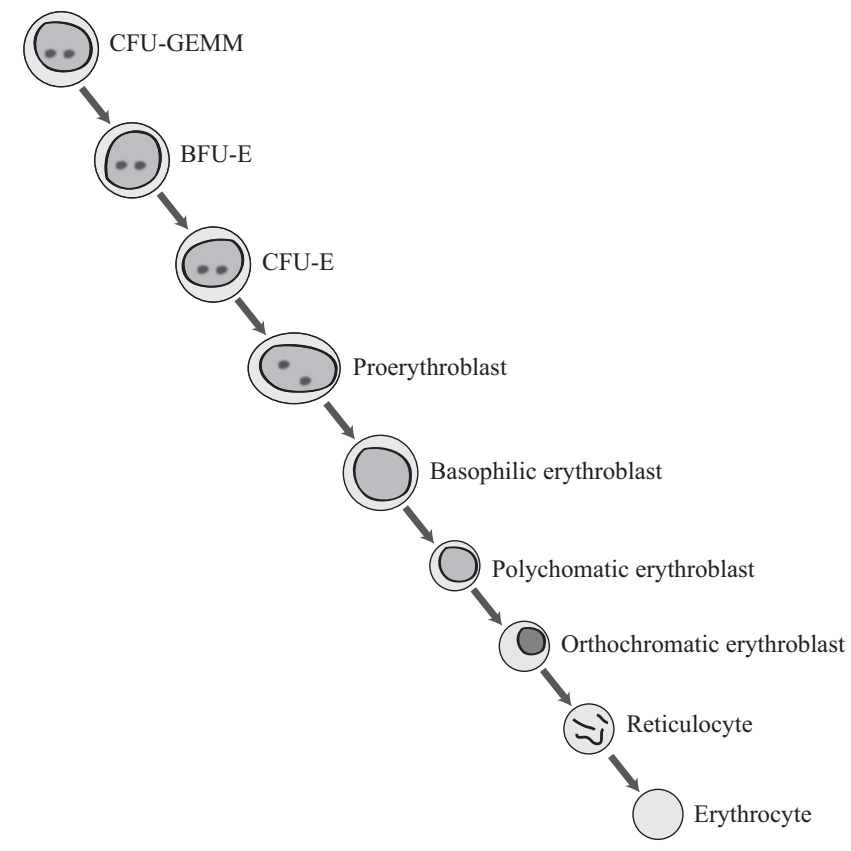

Figure 1: Erythropoiesis cell lineage.

Hemoglobin is synthesized beginning in the basophilic erythrocyte stage, with the majority of synthesis occurring in the polychromatic erythroblast stage. When the nucleus is extruded from the cell, the cell is named a reticulocyte. Little hemoglobin synthesis happens at the reticulocyte stage, and synthesis is completely absent in mature erythrocytes [20]. Reticulocytes begin to lose the adhesive proteins that hold them in the bone marrow. They decrease in size and begin to circulate in the blood. In healthy individuals, erythrocyte life span is approximately 120 days, at which time aging erythrocytes are enveloped by macrophages in the spleen. 
In a healthy individual, an increase in blood oxygen level is detected by the kidneys, which in turn decrease production of EPO. Down-regulation of the RBC production is achieved by the increased death rate of $\mathrm{RBC}$ progenitors due to this decrease in EPO. However, it takes 18 to 21 days for cells to mature and be released into circulation, so this down-regulation has a long delay before it actually affects blood oxygen level. Additionally, down-regulation would only cause the circulating red cell mass to drop by about $1 \%$ per day [32].

Neocytolysis has been observed in astronauts entering space [4, 43] and in high altitude dwellers who descend to sea level [28, 34]; all have higher aggregate RBC mass than needed. Their RBC mass was decreased by $10-15 \%$ in a few days, which could not be explained by simple down-regulation of RBC production.

Neocytolysis, believed to be caused by a drop in EPO level, is a physiological process that aids in this control of the red cell mass by causing the selective destruction of young circulating RBCs $[11,32]$. Since these cells were circulating and contributing to blood oxygen level, their death has a much faster effect on blood oxygen level than the down-regulation of the production cycle. Neocytolysis has been determined to contribute to the anemia of renal disease $[3,33]$, as patients undergoing therapy have constantly fluctuating EPO levels.

\section{$4 \quad$ RBC Model}

Cells in the RBC lineage divide, differentiate and die under the influence of several hormones. Figure 2, a modification of a figure in [22], depicts different aspects of the system we will model. This figure contains possible variables that one might use in formulating mathematical models for red blood cell production. In particular, this figure suggests five cell classes:

- $P_{1}$ : early BFU-E

- $P_{2}$ : late BFU-E, CFU-E, proerythroblasts, and early basophilic erythroblasts

- $P_{3}$ : late basophilic erythroblasts and early polychromatic erythroblasts

- $P_{4}$ : late polychromatic erythroblasts, orthochromatic erythroblasts, and non-circulating reticulocytes

- $P_{5}$ : mature erythrocytes and circulating reticulocytes.

We discuss each compartment individually in Sections 4.2 through 4.6, but it should be noted that the original system chosen to name the erythroid cell progression was based on distinguishing characteristics when samples were taken, stained, and viewed through a microscope. It is not surprising that we choose our five classes of cells differently as we focus on the interactions with EPO and iron and the location of the cells (in the bone marrow or circulating).

The current RBC lineage model is represented in Figure 3. We introduce the model for the iron compartments in Section 5. 


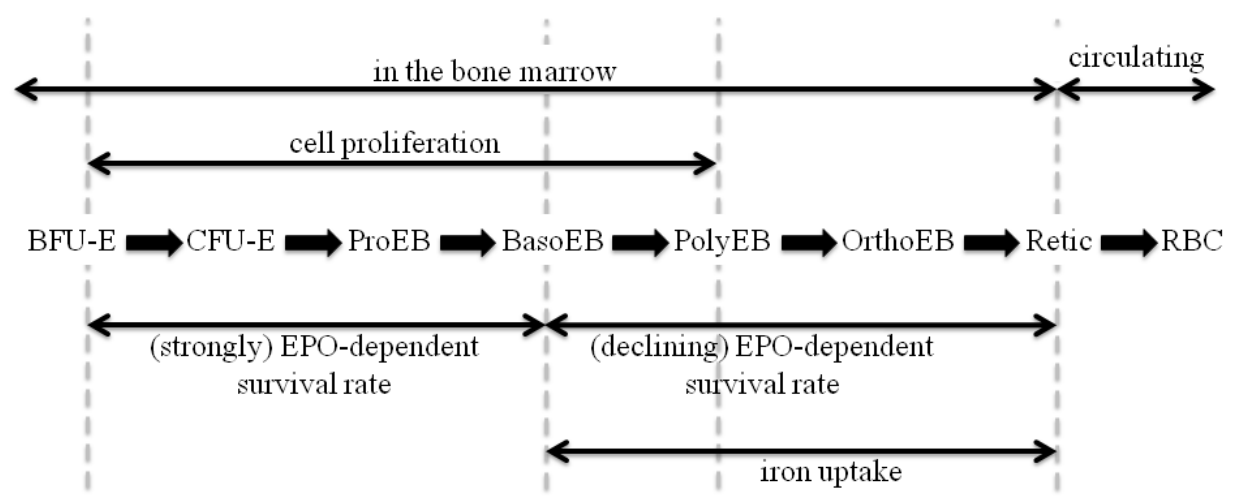

Figure 2: Stages of red blood cell production. We use the following short-hand notations: BFU-E = burst-forming unit-erythroid, CFU-E = colony-forming unit-erythroid, ProEB = proerythroblast, BasoEB = basophilic erythroblast, PolyEB = polychromatic erythroblast, OrthoEB $=$ orthochromatic erythroblast, Retic $=$ reticulocyte, $\mathrm{RBC}=$ red blood cell .

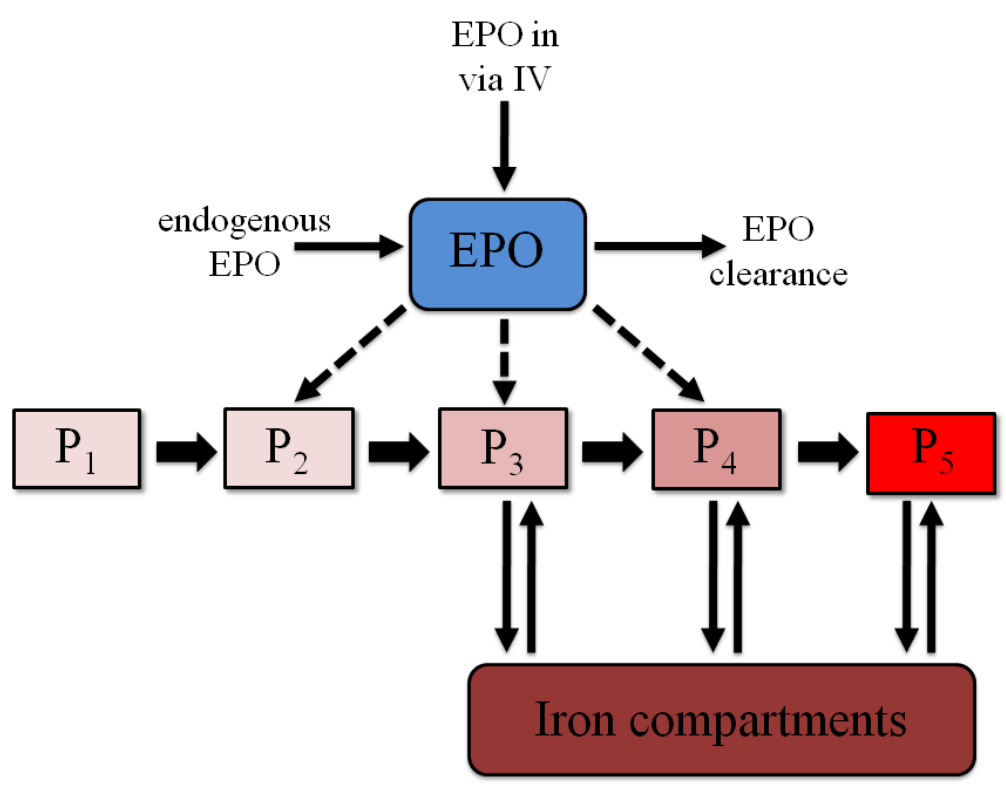

Figure 3: Model schematic. Note that the states variables are $P_{1}\left(t, \mu_{1}\right), P_{2}\left(t, \mu_{2}\right), P_{3}\left(t, \mu_{3}, \gamma\right)$, $P_{4}\left(t, \mu_{4}, \gamma\right), P_{5}\left(t, \mu_{5}, \gamma\right)$ and $E P O(t)$. The iron model is described in Section 5 . 


\subsection{Derivation of Model Equations}

We use a model with two structure variables, one for maturity level and one for cellular iron level. The structure variables $\mu_{i}$ and $\gamma$ are unitless and represent the maturity level and iron state, respectively, of cells. Thus, $P_{3}\left(t, \mu_{3}, \gamma\right)$ is the number of cells (in billions) in population $P_{3}$ at time $t$ that have maturity level $\mu_{3}$ and iron state $\gamma$. Each class is assumed to have a maximum maturity level, $\left(\mu_{i}\right)_{f}$.

Throughout, we use $\beta$ to represent a birth/proliferation rate, $\delta$ to represent a death rate, and $\rho$ to represent other rates (maturation rates, rates of treatment, etc.).

We will assume that the iron state structure variable $\gamma$ varies from 0 to $\gamma_{f}=2$, where $\gamma_{\text {full }}=\frac{\gamma_{f}}{2}=1$ represents the amount of iron the "typical" RBC contains in a healthy individual (this quantity is to be estimated later). We will assume that when a cell divides, each daughter cell inherits half of the parent cell's iron. Therefore, when a cell with iron state $\gamma$ divides, it leaves iron state $\gamma$ and two cells enter iron state $\frac{\gamma}{2}$. Similarly, for each cell in iron class $2 \gamma$ that divides, two cells enter iron class $\gamma$.

We derive equations for those classes that incorporate iron by considering, at some time $t$, an incremental "area" of size $\Delta \mu$ by $\Delta \gamma$, with $0 \leq \gamma \leq \frac{\gamma_{f}}{2}=1$, as in Figure 4.

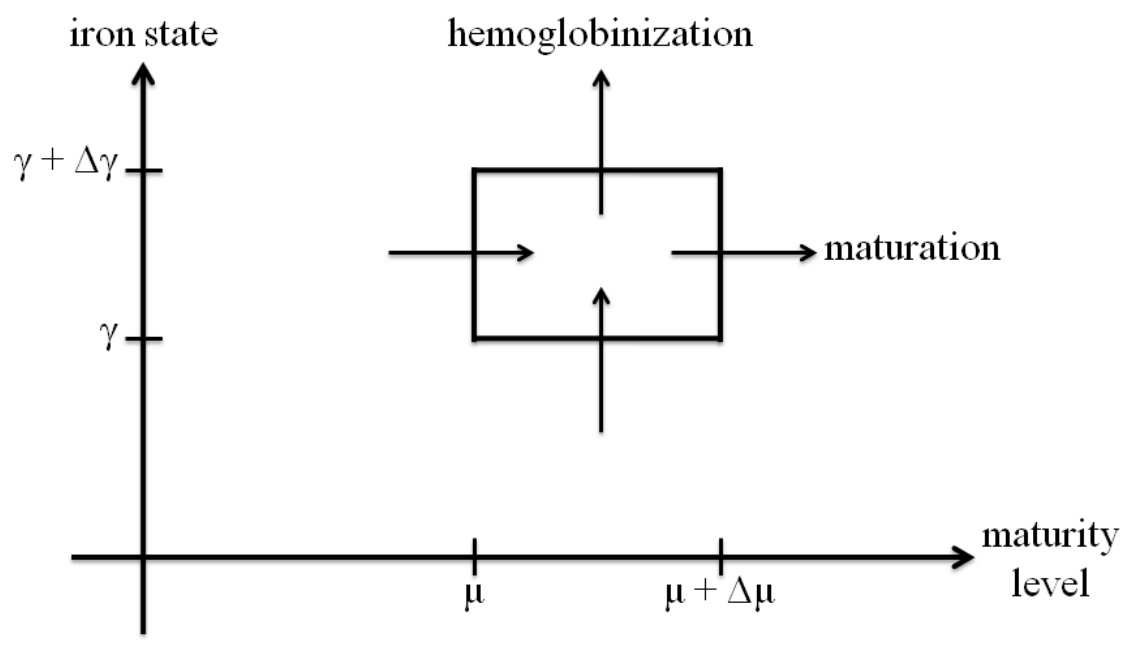

Figure 4: Maturation and hemoglobinization processes.

We treat this region as a arbitrary elemental compartment and consider the time rate of change of the cell population in the compartment. 


$$
\begin{aligned}
\frac{\partial}{\partial t} \int_{\gamma}^{\gamma+\Delta \gamma} \int_{\mu}^{\mu+\Delta \mu} P(t, \xi, \zeta) d \xi d \zeta= & 2 \text { (rate entering iron class } \gamma \text { from class } 2 \gamma) \\
& -(\text { rate of cells leaving iron class } \gamma) \\
& -(\text { death rate })+(\text { rate of maturation in }) \\
& -(\text { rate of maturation out }) \\
& +(\text { rate of hemoglobinization in }) \\
& -(\text { rate of hemoglobinization out }) \\
=2 \int_{\gamma}^{\gamma+\Delta \gamma} \int_{\mu}^{\mu+\Delta \mu} \beta P(t, \xi, 2 \zeta) d \xi d \zeta & \\
& -\int_{\gamma}^{\gamma+\Delta \gamma} \int_{\mu}^{\mu+\Delta \mu} \beta P(t, \xi, \zeta) d \xi d \zeta \\
& -\int_{\gamma}^{\gamma+\Delta \gamma} \int_{\mu}^{\mu+\Delta \mu} \delta P(t, \xi, \zeta) d \xi d \zeta \\
& +\int_{\gamma}^{\gamma+\Delta \gamma} \rho P(t, \mu, \zeta) d \zeta-\int_{\gamma}^{\gamma+\Delta \gamma} \rho P(t, \mu+\Delta \mu, \zeta) d \zeta \\
& +\int_{\mu}^{\mu+\Delta \mu} h P(t, \xi, \gamma) d \xi-\int_{\mu}^{\mu+\Delta \mu} h P(t, \xi, \gamma+\Delta \gamma) d \xi,
\end{aligned}
$$

where $0 \leq \mu \leq \mu_{f}$, $\beta$ is the proliferation rate, $\delta$ is the death rate, $\rho$ is the maturation rate, and $h$ is the hemoglobinization rate (equivalent to the rate of iron uptake), which generally depends on the plasma iron level $F e_{p l}$.

We rearrange these to obtain

$$
\begin{aligned}
\frac{\partial}{\partial t} \int_{\gamma}^{\gamma+\Delta \gamma} \int_{\mu}^{\mu+\Delta \mu} P(t, \xi, \zeta) d \xi d \zeta=2 & \int_{\gamma}^{\gamma+\Delta \gamma} \int_{\mu}^{\mu+\Delta \mu} \beta P(t, \xi, 2 \zeta) d \xi d \zeta \\
& -\int_{\gamma}^{\gamma+\Delta \gamma} \int_{\mu}^{\mu+\Delta \mu}[\beta+\delta] P(t, \xi, \zeta) d \xi d \zeta \\
& -\int_{\gamma}^{\gamma+\Delta \gamma} \rho[P(t, \mu+\Delta \mu, \zeta)-P(t, \mu, \zeta)] d \zeta \\
& -\int_{\mu}^{\mu+\Delta \mu} h[P(t, \xi, \gamma+\Delta \gamma)-P(t, \xi, \gamma)] d \xi
\end{aligned}
$$


then divide by $\Delta \mu \Delta \gamma$ :

$$
\begin{aligned}
\frac{\partial}{\partial t}\left[\frac{1}{\Delta \gamma} \int_{\gamma}^{\gamma+\Delta \gamma} \frac{1}{\Delta \mu} \int_{\mu}^{\mu+\Delta \mu} P(t, \xi, \zeta) d \xi d \zeta\right]= & \frac{2}{\Delta \gamma} \int_{\gamma}^{\gamma+\Delta \gamma} \frac{1}{\Delta \mu} \int_{\mu}^{\mu+\Delta \mu} \beta P(t, \xi, 2 \zeta) d \xi d \zeta \\
& -\frac{1}{\Delta \gamma} \int_{\gamma}^{\gamma+\Delta \gamma} \frac{1}{\Delta \mu} \int_{\mu}^{\mu+\Delta \mu}[\beta+\delta] P(t, \xi, \zeta) d \xi d \zeta \\
& -\frac{1}{\Delta \gamma} \int_{\gamma}^{\gamma+\Delta \gamma} \rho \frac{P(t, \mu+\Delta \mu, \zeta)-P(t, \mu, \zeta)}{\Delta \mu} d \zeta \\
& -\frac{1}{\Delta \mu} \int_{\mu}^{\mu+\Delta \mu} h \frac{P(t, \xi, \gamma+\Delta \gamma)-P(t, \xi, \gamma)}{\Delta \gamma} d \xi .
\end{aligned}
$$

When we allow $\Delta \mu \rightarrow 0$ and $\Delta \gamma \rightarrow 0$, we obtain

$$
\begin{array}{cc}
\frac{\partial}{\partial t} P(t, \mu, \gamma)=2 \beta P(t, \mu, 2 \gamma)-[\beta+\delta] P(t, \mu, \gamma)-\frac{\partial}{\partial \mu} \rho P(t, \mu, \gamma) & \\
-\frac{\partial}{\partial \gamma} h P(t, \mu, \gamma), & 0 \leq \gamma \leq \frac{\gamma_{f}}{2}=1,
\end{array}
$$

the general form of the equations governing cells in classes $P_{3}$ through $P_{5}$. Note that the rates $\beta, \delta, \rho$ and $h$ could be functions that depend on $\mu, \gamma$ or other states in the system.

By our assumptions, the maximum iron state is $\gamma_{f}=2$, so cell division cannot yield a cell with iron state $\gamma>\frac{\gamma_{f}}{2}=1$. Thus, for cells with iron states greater than $\frac{\gamma_{f}}{2}=1$, we omit the birth rate term associated with iron state $2 \gamma$, as below:

$$
\frac{\partial}{\partial t} P(t, \mu, \gamma)=-[\beta+\delta] P(t, \mu, \gamma)-\frac{\partial}{\partial \mu} \rho P(t, \mu, \gamma)-\frac{\partial}{\partial \gamma} h P(t, \mu, \gamma), \quad \frac{\gamma_{f}}{2}=1 \leq \gamma \leq \gamma_{f}=2 .
$$

Thus our generic balance laws for the $P_{3}$ through $P_{5}$ compartments can be written (using the characteristic function $\chi$ for the interval $[0,1]$, i.e., $\chi_{[0,1]}(\gamma)=1$ when $\gamma \in[0,1]$ and $\chi(\gamma)=0$ otherwise) as

$$
\begin{array}{rlrl}
\frac{\partial}{\partial t} P(t, \mu, \gamma)=\chi_{[0,1]}(\gamma) & 2 \beta P(t, \mu, 2 \gamma)-[\beta+\delta] P(t, \mu, \gamma)-\frac{\partial}{\partial \mu} \rho P(t, \mu, \gamma) & \\
- & \frac{\partial}{\partial \gamma} h P(t, \mu, \gamma), & 0 & \leq \gamma \leq 2 .
\end{array}
$$

Cells in classes $P_{1}\left(t, \mu_{1}\right)$ and $P_{2}\left(t, \mu_{2}\right)$ do not incorporate iron, so they are functions of only time and maturity level. The general form of the partial differential equations governing these classes, derived in [5], is

$$
\frac{\partial}{\partial t} P(t, \mu)=[\beta-\delta] P(t, \mu)-\frac{\partial}{\partial \mu} \rho P(t, \mu),
$$

where $\beta$ is the proliferation rate, $\delta$ is the death rate, and $\rho$ is the maturation rate. 


\subsection{Class $P_{1}\left(t, \mu_{1}\right)$}

Class $P_{1}$ consists of early BFU-E. These are the most immature cells that are committed to the erythroid lineage. We make the following assumptions about cells in this class.

1. The maturation rate is constant, $\rho_{1}$.

2. BFU-E differentiate into CFU-E in approximately seven days [35, 38]. BFU-E begin expressing EPO receptors (EPORs), which means that, in time, they do become influenced by EPO. Hence, we allow for cells to reside in class $P_{1}$ for three days (during which they are not influenced by EPO), while late BFU-E are in class $P_{2}$ (where they are under the influence of EPO). Therefore we choose the maximum maturity level to be $\left(\mu_{1}\right)_{f}=3$.

3. The majority of cell proliferation happens in later classes $[22,23]$, so we set birth rate equal to death rate for cells in this class.

Hence, for class $P_{1}$, equation (4) becomes

$$
\frac{\partial}{\partial t} P_{1}\left(t, \mu_{1}\right)=-\rho_{1} \frac{\partial}{\partial \mu_{1}} P_{1}\left(t, \mu_{1}\right)
$$

with initial condition

$$
P_{1}\left(0, \mu_{1}\right)=P_{1}^{\text {init }}\left(\mu_{1}\right)
$$

and boundary condition at $\mu_{1}=0$

$$
P_{1}(t, 0)=P_{1}^{b d y}(t)
$$

\subsection{Class $P_{2}\left(t, \mu_{2}\right)$}

Class $P_{2}$ consists of late BFU-E, CFU-E, proerythroblasts, and early basophilic erythroblasts. We make the following assumptions about cells in class $P_{2}$.

1. The maturation rate is constant, $\rho_{2}$.

2. Cells reside in this class for twelve days [17, 35], and therefore $\left(\mu_{2}\right)_{f}=12$.

3. During the time cells reside in class $P_{2}$, they undergo approximately 6 cell divisions $[17,18,19,22]$. We assume that the birth rate is a constant, $\beta_{2}$.

4. Cells in this class express EPORs [23, 37], and this interaction of EPO with EPOR is the most important control point for erythropoiesis [17, 36, 47]. Cells at this stage depend absolutely on EPO for their survival and will undergo apoptosis in its absence [23, 38]. Even in healthy individuals, complete survival of progenitors would require an EPO level much higher than normal level; the normal production rate of RBCs represents survival of only a minority of progenitor cells [22]. Therefore we choose the death rate to be a decreasing function of the EPO level:

$$
\delta_{2}(E P O)=\left(\delta_{2}^{\text {max }}-\delta_{2}^{\text {min }}\right) \cdot \frac{\left(c_{\delta_{2}}\right)^{k_{\delta_{2}}}}{\left(c_{\delta_{2}}\right)^{k_{\delta_{2}}}+(E P O)^{k_{\delta_{2}}}}+\delta_{2}^{\text {min }} .
$$


Thus, using equation (4), the state equation for class $P_{2}$ is given by

$$
\frac{\partial}{\partial t} P_{2}\left(t, \mu_{2}\right)=\left[\beta_{2}-\delta_{2}(E P O)\right] P_{2}\left(t, \mu_{2}\right)-\rho_{2} \frac{\partial}{\partial \mu_{2}} P_{2}\left(t, \mu_{2}\right),
$$

with initial condition

$$
P_{2}\left(0, \mu_{2}\right)=P_{2}^{\text {init }}\left(\mu_{2}\right)
$$

and boundary condition at $\left(\mu_{1}\right)_{f}=3, \mu_{2}=0$

$$
P_{2}(t, 0)=P_{1}(t, 3) \text {. }
$$

\subsection{Class $P_{3}\left(t, \mu_{3}, \gamma\right)$}

Class $P_{3}$ consists of late basophilic erythroblasts and early polychromatic erythroblasts. We assume the following.

1. The maturation rate is constant, $\rho_{3}$.

2. Cells reside in this class for 1 day $[17,35]$, and therefore $\left(\mu_{3}\right)_{f}=1$.

3. Cells in this stage continue to proliferate [17], with a constant rate of $\beta_{3}=\ln 2$ days $^{-1}$, which corresponds to one cell division per day.

4. Cells in class $P_{3}$ continue to express EPORs, but the level of expression declines significantly as cells mature through this class [12]. Hence, cells in this class become less dependent on EPO for survival. We model this as

$$
\delta_{3}\left(\mu_{3}, E P O\right)=\delta_{3}^{\max }\left(\mu_{3}\right) \cdot \frac{\left(c_{\delta_{3}}\right)^{k_{\delta_{3}}}}{\left(c_{\delta_{3}}\right)^{k_{\delta_{3}}}+(E P O)^{k_{\delta_{3}}}}
$$

where $\delta_{3}^{\max }\left(\mu_{3}\right)$ is a decreasing function, which we assume to be affine for this model given by

$$
\delta_{3}^{\max }\left(\mu_{3}\right)=-m_{3} \mu_{3}+b_{3}, \quad \text { where } m_{3}>0, \quad b_{3}-m_{3} \cdot\left(\mu_{3}\right)_{f}=0 .
$$

The function $\delta_{3}\left(\mu_{3}, E P O\right)$, for a fixed maturity level $\mu_{3}$, is a decreasing sigmoid function of EPO. That is, at a fixed maturity level, as EPO increases, the death rate decreases. If we consider instead a fixed EPO level, then increasing maturation level causes a decrease in the maximum death rate. That is, low EPO affects younger cells in this class more than it affects more mature cells. Both the fixed maturity level and fixed EPO level phenomena are depicted in Figure 5.

5. Cells in class $P_{3}$ express transferrin receptors (Tfr) and begin the process of taking in iron and synthesizing hemoglobin [17, 22]. The rate of hemoglobinization (or equivalently iron uptake) is a function of iron level $F e_{p l}$ (in the blood plasma) and iron state $\gamma$ (of a cell). A full description of the hemoglobinization rate, $h_{3}\left(F e_{p l}, \gamma\right)$, along with definition of the parameter $\mathrm{Fe}_{p l}$ appears in Section 4.7. 
Class $\mathrm{P}_{3}$ death rate versus maturity level

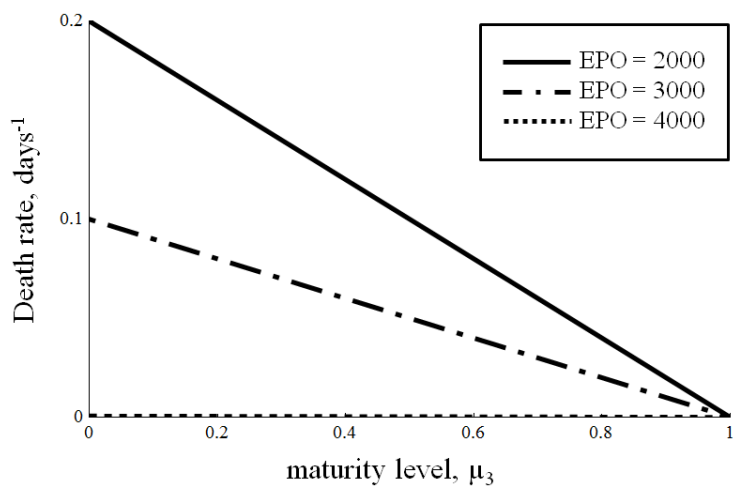

Class $\mathrm{P}_{3}$ death rate versus EPO level

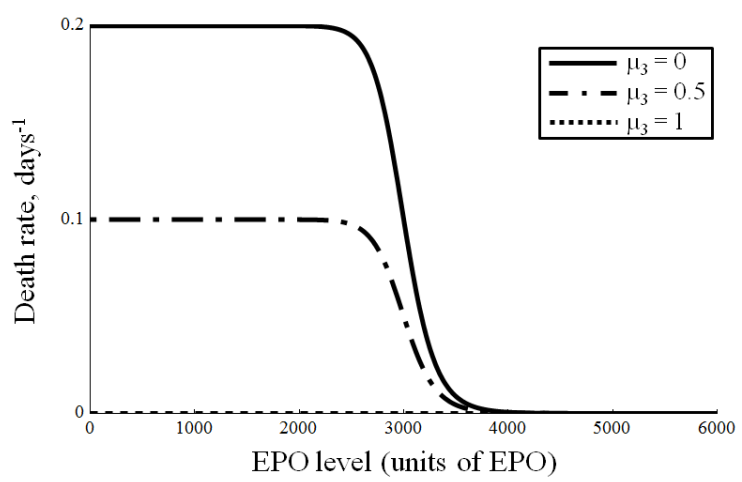

Figure 5: Death rate in class $P_{3}$ is a function of both maturity level, $\mu_{3}$, and EPO level. For a fixed EPO level, death rate decreases as maturity level increases. For a fixed maturity level, death rate is larger when EPO is small. These plots were generated with parameter values $m_{3}=0.2, b_{3}=0.2, c_{\delta_{3}}=3000$ and $k_{\delta_{3}}=20$.

Hence, for class $P_{3}$, equation (3) becomes

$$
\begin{array}{cc}
\frac{\partial}{\partial t} P_{3}\left(t, \mu_{3}, \gamma\right)=\chi_{[0,1]}(\gamma) 2 \beta_{3} P_{3}\left(t, \mu_{3}, 2 \gamma\right)-\left[\beta_{3}+\delta_{3}\left(\mu_{3}, E P O\right)\right] P_{3}\left(t, \mu_{3}, \gamma\right)-\rho_{3} \frac{\partial}{\partial \mu_{3}} P_{3}\left(t, \mu_{3}, \gamma\right) \\
-\frac{\partial}{\partial \gamma}\left[h_{3}\left(F e_{p l}, \gamma\right) P_{3}\left(t, \mu_{3}, \gamma\right)\right], & 0 \leq \gamma \leq 2
\end{array}
$$

with initial condition

$$
P_{3}\left(0, \mu_{3}, \gamma\right)=P_{3}^{\text {init }}\left(\mu_{3}, \gamma\right)
$$

and boundary conditions at $\left(\mu_{2}\right)_{f}=12, \mu_{3}=0$

$$
P_{3}(t, 0, \gamma)= \begin{cases}P_{2}(t, 12), & \gamma=0 \\ 0, & \gamma \neq 0\end{cases}
$$

and

$$
P_{3}\left(t, \mu_{3}, 0\right)= \begin{cases}P_{2}(t, 12), & \mu_{3}=0 \\ P_{3}^{b d y, \gamma}\left(t, \mu_{3}\right), & \mu_{3} \neq 0\end{cases}
$$

\subsection{Class $P_{4}\left(t, \mu_{4}, \gamma\right)$}

Class $P_{4}$ contains late polychromatic erythroblasts, orthochromatic erythroblasts, and noncirculating reticulocytes, the last stages of erythroid cells residing in the bone marrow. We make the following assumptions about cells in class $P_{4}$. 
1. The maturation rate is constant, $\rho_{4}$.

2. Cells reside in this class for 2 days $[17,35]$, and therefore $\left(\mu_{4}\right)_{f}=2$.

3. Cells in this class have stopped proliferating $[17,22]$. Thus, $\beta_{4}=0$.

4. It is well-documented that red blood cells have a shorter life span in individuals with iron deficiency $[10,13,14,44]$. Hence, we assume that any RBCs in class $P_{4}$ that are severely iron deficient have increased mortality.

We begin by defining $c_{\delta_{4}}\left(\mu_{4}\right)$ as an increasing function of $\mu_{4}$. For simplicity, we choose an affine function,

$$
c_{\delta_{4}}\left(\mu_{4}\right)=m_{4} \mu_{4}+b_{4}, \quad m_{4}>0, \quad b_{4} \geq 0,
$$

with the added restriction that $0<c_{\delta_{4}}\left(\mu_{4}\right)<\left(\mu_{4}\right)_{f}$ for all $\mu_{4}$.

We assume now that death rate depends on maturity level and cellular iron state, as below :

$$
\delta_{4}\left(\mu_{4}, \gamma\right)=\left(\delta_{4}^{\max }-\delta_{4}^{\min }\right) \cdot \frac{\left(c_{\delta_{4}}\left(\mu_{4}\right)\right)^{k_{\delta_{4}}}}{\left(c_{\delta_{4}}\left(\mu_{4}\right)\right)^{k_{\delta_{4}}}+(\gamma)^{k_{\delta_{4}}}}+\delta_{4}^{\text {min }} .
$$

Notice that when $\gamma$ is relatively small, $\delta_{4}\left(\mu_{4}, \gamma\right)$ is close to $\delta_{4}^{\max }$, and when $\gamma$ is relatively large, $\delta_{4}\left(\mu_{4}, \gamma\right)$ is close to $\delta_{4}^{\text {min }}$. However, the "relativity" is affected by $c_{\delta_{4}}\left(\mu_{4}\right)$ when $\gamma<\gamma_{c r i t, 4}$. As cells mature through class $P_{4}$ (i.e., as $\mu_{4}$ increases), then $c_{\delta_{4}}\left(\mu_{4}\right)$ increases, which means that the death rate for a given iron level $\gamma$ also increases (we choose $k_{\delta_{4}}>1$ ). Hence, a cell having only a little iron when it arrives in class $P_{4}$ is less likely to die than a cell with that same level of iron that is about to mature out of class $P_{4}$. Figure 6 depicts $\delta_{4}\left(\mu_{4}, \gamma\right)$ over varying $\mu_{4}$ and $\gamma$.

5. Cells in class $P_{4}$ continue to collect iron and synthesize hemoglobin $[17,22]$ at a rate $h_{4}\left(F e_{p l}, \gamma\right)$, explained further in Section 4.7.

Thus, using equation (3), the state equation is given by

$$
\begin{aligned}
\frac{\partial}{\partial t} P_{4}\left(t, \mu_{4}, \gamma\right)=- & \delta_{4}\left(\mu_{4}, \gamma\right) P_{4}\left(t, \mu_{4}, \gamma\right)-\rho_{4} \frac{\partial}{\partial \mu_{4}} P_{4}\left(t, \mu_{4}, \gamma\right) \\
& -\frac{\partial}{\partial \gamma}\left[h_{4}\left(F e_{p l}, \gamma\right) P_{4}\left(t, \mu_{4}, \gamma\right)\right]
\end{aligned}
$$

with initial condition

$$
P_{4}\left(0, \mu_{4}, \gamma\right)=P_{4}^{\text {init }}\left(\mu_{4}, \gamma\right)
$$

and boundary conditions at $\left(\mu_{3}\right)_{f}=1, \mu_{4}=0$

$$
P_{4}(t, 0, \gamma)=P_{3}(t, 1, \gamma)
$$

and

$$
P_{4}\left(t, \mu_{4}, 0\right)= \begin{cases}P_{3}(t, 1,0), & \mu_{4}=0 \\ P_{4}^{b d y, \gamma}\left(t, \mu_{4}\right), & \mu_{4} \neq 0\end{cases}
$$


Class $\mathrm{P}_{4}$ death rate versus maturity level

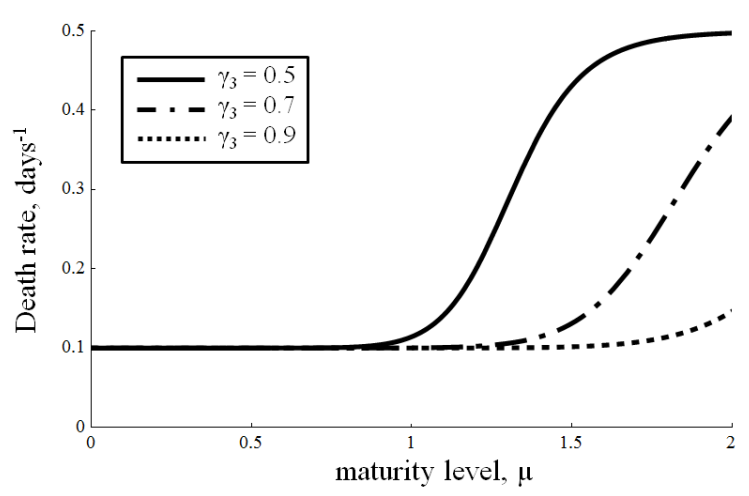

Class $\mathrm{P}_{4}$ death rate versus iron state

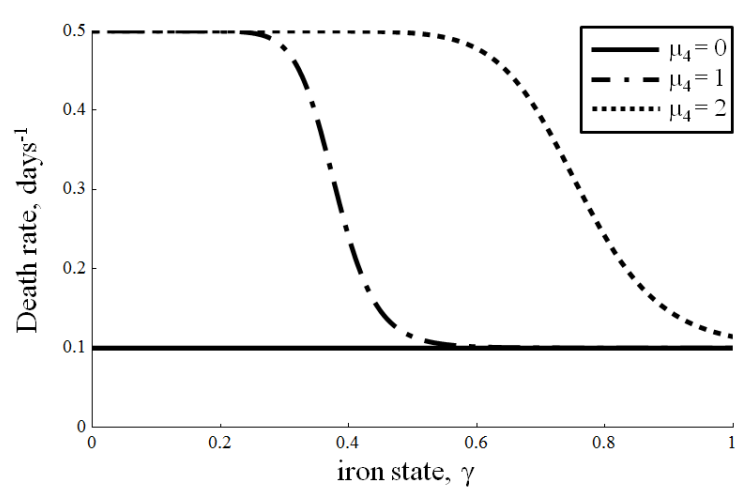

Figure 6: Death rate in class $P_{4}$ is a function of both maturity level, $\mu_{4}$, and cellular iron state, $\gamma$. For a fixed maturity level $\mu_{4}$, death rate decreases as iron state $\gamma$ increases. That is, for a given maturity level, cells with little iron are more likely to die. For a iron state $\gamma<\gamma_{c r i t, 4}$, death rate is increases as maturity level increases. These plots were generated with parameter values $\delta_{4}^{\min }=0.1, \delta_{4}^{\max }=0.5, m_{4}=0.38, b_{4}=0$, and $k_{\delta_{4}}=12$.

\subsection{Class $P_{5}\left(t, \mu_{5}, \gamma\right)$}

Cells in class $P_{5}$ are mature erythrocytes and circulating reticulocytes. For cells in this class, we make the following assumptions.

1. The maturation rate is constant, $\rho_{5}$.

2. The average life span of a red blood cell in a patient with chronic kidney disease is about 70 days, significantly shorter than for healthy persons [24, 39, 41]. We set the maximum maturity level to be $\left(\mu_{5}\right)_{f}=100$ days $^{-1}$, but the death rate is set such that virtually no cells reach the maximum maturity level.

3. As they have no nuclei, cells in this stage do not proliferate [17], and therefore $\beta_{5}=0$.

4. Death rate in this class is assumed to have four components: death due to low cellular iron, death due to aging, death due to neocytolysis, and death due to blood loss.

(a) Death due to low cellular iron. As in the previous class, cells that lack a full complement of iron have increased mortality. As in class $P_{4}$, we have

$$
c_{\delta_{5, \gamma}}\left(\mu_{5}\right)=m_{5} \mu_{5}+b_{5}, \quad m_{5}>0,
$$


and

$$
\delta_{5, \gamma}\left(\mu_{5}, \gamma\right)=\left(\delta_{5, \gamma}^{\max }-\delta_{5, \gamma}^{\min }\right) \cdot \frac{\left(c_{\delta_{5, \gamma}}\left(\mu_{5}\right)\right)^{k_{\delta_{5, \gamma}}}}{\left(c_{\delta_{5, \gamma}}\left(\mu_{5}\right)\right)^{k_{\delta_{5, \gamma}}}+(\gamma)^{k_{\delta_{5, \gamma}}}}+\delta_{5, \gamma}^{\min } .
$$

(b) Death due to aging. Senescent (aged) erythrocytes are enveloped and destroyed in the spleen [17], so death rate in this class is also a function of maturity level. This process occurs independent of cellular iron state:

$$
\delta_{5, \mu}\left(\mu_{5}\right)=\left(\delta_{\mu_{5}}^{\max }-\delta_{\mu_{5}}^{\min }\right) \cdot \frac{\left(c_{\delta_{\mu_{5}}}\right)^{k_{\delta_{\mu_{5}}}}}{\left(c_{\delta_{\mu_{5}}}\right)^{k_{\mu_{5}}}+\left(\mu_{5}\right)^{k_{\delta_{\mu_{5}}}}}+\delta_{\mu_{5}}^{\text {min }}
$$

(c) Death due to neocytolysis. Neocytolysis targets RBCs between 14-21 days old (i.e., for maturity level $\left.14 \leq \mu_{5} \leq 21\right)[4,32]$. These cells have increased death rate when EPO level drops, but it is not yet known whether the mechanisms that cause this increase in death rate are related to a large drop in EPO level (i.e., a large negative rate of change of EPO level) and/or simply a low EPO level. We account for both in this model, with tuning parameters $\alpha_{1}$ and $\alpha_{2}$ to account for the relative effects.

We begin with the assumption that neocytolysis is a response to low EPO level. We choose a decreasing sigmoid function for the maximum death rate:

$$
\delta_{5, \text { neo }, 1}^{\max }(E P O)=\delta_{5, \text { neo }}^{\max , 1} \cdot \frac{\left(c_{\delta_{5}}^{\text {neo }}\right)^{k_{\delta_{5}}^{\text {neo }}}}{\left(c_{\delta_{5}}^{\text {neo }}\right)^{k_{\delta_{5}}^{\text {neo }}}+(E P O)^{k_{\delta_{5}}^{\text {neo }}}} .
$$

Then the death rate is given by

$$
\delta_{5, \text { neo }, 1}\left(\mu_{5}, E P O\right)=\delta_{5, \text { neo }, 1}^{\max }(E P O)\left[\frac{1}{1+e^{-2 k_{h, 1}\left(\mu_{5}-15\right)}}-\frac{1}{1+e^{-2 k_{h, 1}\left(\mu_{5}-20\right)}}\right],
$$

the sum of smoothed heaviside functions. Note that when $k_{h, 1}$ is chosen large enough, the death rate is zero when $\mu_{5}<14$ and $\mu_{5}>21$. Also, for maturity levels $14 \leq \mu_{5} \leq 21$, when EPO level is small, the maximum death rate is large. Figure 7 depicts the output of the death rate function $\delta_{5, \text { neo, } 1}\left(\mu_{5}, E P O\right)$, for both the case of a fixed maturity level with varying EPO and the case of fixed EPO with varying maturity level.

For the assumption that neocytolysis is a response to large negative rate of change of EPO, we choose a decreasing function for the maximum death rate:

$$
\delta_{5, \text { neo }, 2}^{\max }\left(\frac{d}{d t} E P O\right)=\delta_{5, \text { neo }}^{\max , 2}\left[1-\frac{1}{1+e^{-2 k_{h, 3}\left(\frac{d}{d t} E P O-\rho_{E P O, \text { crit }}\right)}} \cdot\right]
$$

(Note here that we do not use a sigmoid function (as we did for $\delta_{5, \text { neo }, 1}^{\max }(E P O)$ ) because the area of interest includes negative values of $\frac{d}{d t} E P O$.) The death rate, 

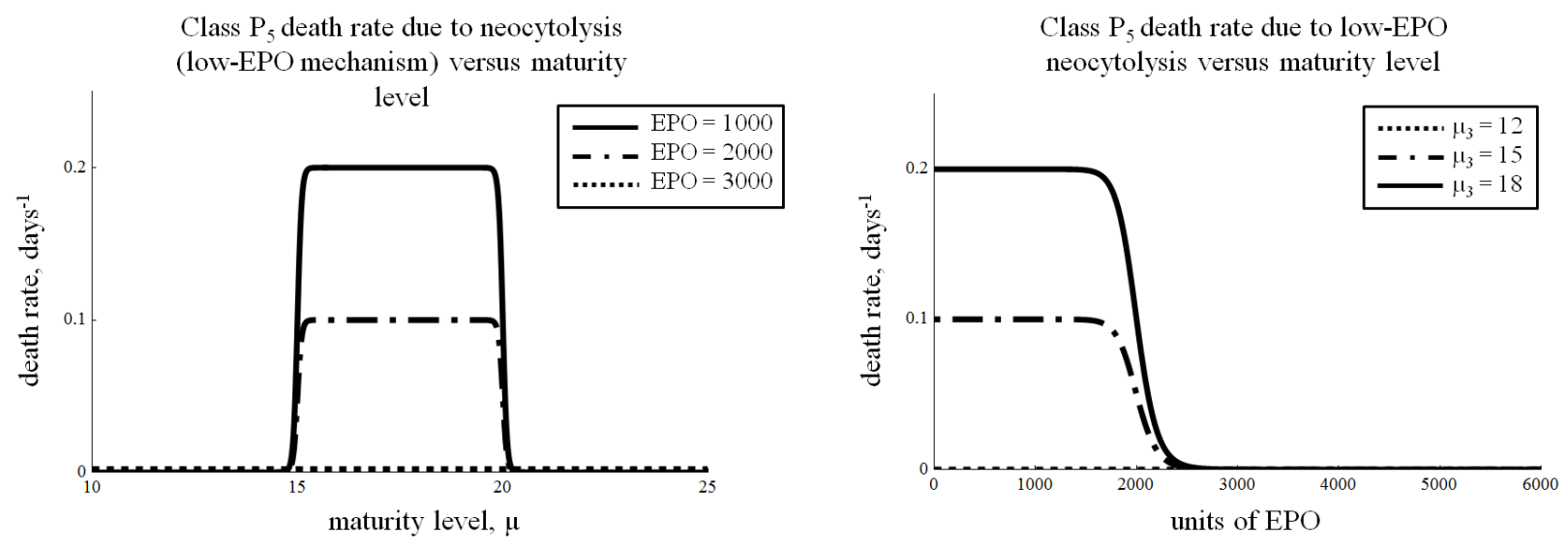

Figure 7: Low-EPO neocytolysis death rate in class $P_{5}$ is a function of maturity level $\mu_{5}$ and EPO level. In the first plot, we fix EPO and show the death rate over varying maturity level. Notice that neocytolysis affects cells such that $14 \leq \mu_{5} \leq 21$. Also, as EPO increases, the effects of neocytolysis diminish. This fact is demonstrated again in the second plot. To produce these plots, we chose parameter values $\delta_{5, \text { neo }}^{\max , 1}=0.2, c_{\delta_{5}}^{\text {neo }}=2000, k_{\delta_{5}}^{\text {neo }}=20$ and $k_{h, 1}=10$.

given by

$$
\begin{aligned}
\delta_{5, \text { пео }, 2} & \left(\mu_{5}, \frac{d}{d t} E P O\right) \\
& =\delta_{5, \text { neo }, 2}^{\max }\left(\frac{d}{d t} E P O\right)\left[\frac{1}{1+e^{-2 k_{h, 2}\left(\mu_{5}-15\right)}}-\frac{1}{1+e^{-2 k_{h, 2}\left(\mu_{5}-20\right)}}\right],
\end{aligned}
$$

is plotted in Figure 8 for both the constant EPO rate with varying maturity case and the constant maturity level with varying EPO rate case.

Then the death rate due to neocytolysis is given by

$$
\delta_{5, \text { пео }}\left(\mu_{5}, E P O, \frac{d}{d t} E P O\right)=\alpha_{1} \delta_{5, \text { пео }, 1}\left(\mu_{5}, E P O\right)+\alpha_{2} \delta_{5, \text { пео }, 2}\left(\mu_{5}, \frac{d}{d t} E P O\right) .
$$

(d) Death due to blood loss. We make a separate death rate to account for losses such as blood lost during blood draws, denoted $\delta_{5, \text { loss }}\left(t, \mu_{5}\right)$. The iron from these losses is not recycled. 

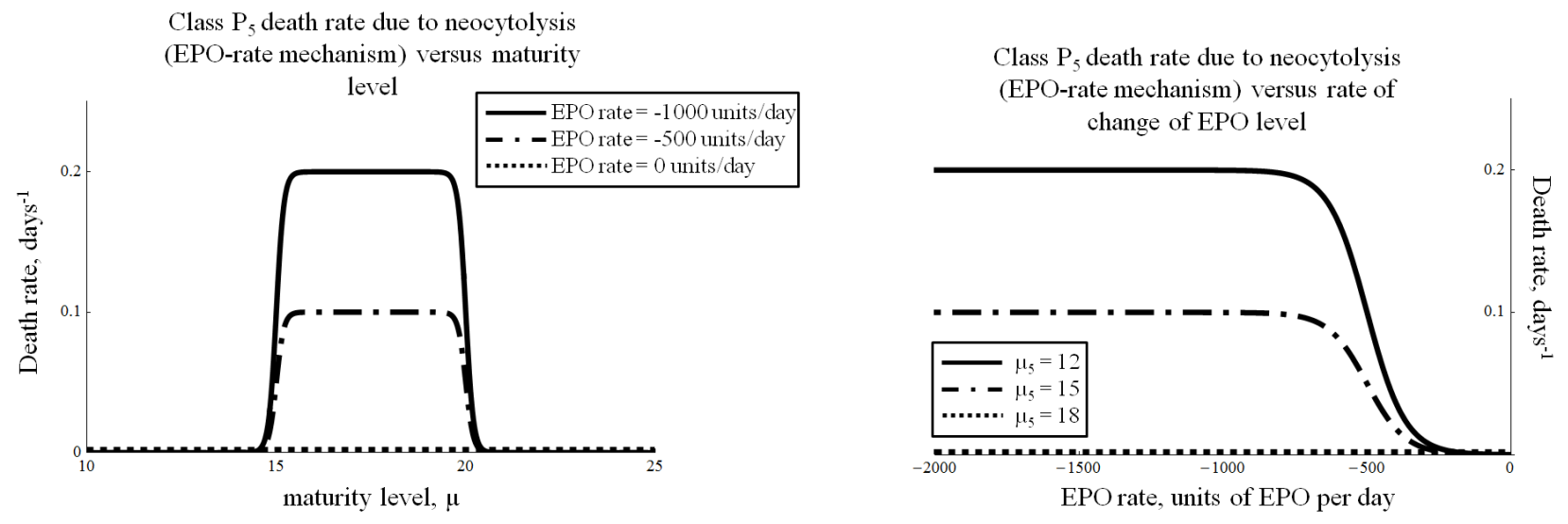

Figure 8: Large negative EPO rate neocytolysis death rate in class $P_{5}$ is a function of maturity level $\mu_{5}$ and EPO rate. Note that neocytolysis targets cells with maturity level $14 \leq \mu_{5} \leq 21$. As EPO rate attains large negative values, the death rate due to neocytolysis increases. To produce these plots, we chose parameter values $\delta_{5, \text { neo }}^{\max , 2}=0.2, k_{h, 3}=0.0075, \rho_{E P O, \text { crit }}=750$ and $k_{h, 2}=5$.

We assume that these phenomena occur independently and that the resultant death rate for class $P_{5}$ is the sum of these four components,

$$
\begin{aligned}
\delta_{5}\left(\mu_{5}, \gamma, E P O, \frac{d}{d t} E P O\right)=\delta_{5, \gamma}\left(\mu_{5}, \gamma\right) & +\delta_{5, \mu}\left(\mu_{5}\right) \\
& +\delta_{5, \text { nео }}\left(\mu_{5}, E P O, \frac{d}{d t} E P O\right)+\delta_{5, \text { loss }}\left(t, \mu_{5}\right) .
\end{aligned}
$$

5. Only reticulocytes at this stage are still able to collect iron, and at a smaller rate than previous cell classes $[9,15,17]$. As before, the hemoglobinization rate, $h_{5}\left(F e_{p l}, \gamma\right)$, for cells in class $P_{5}$ is discussed in Section 4.7 .

Using equation (3), cells in class $P_{5}$ are governed by the equation

$$
\begin{aligned}
\frac{\partial}{\partial t} P_{5}\left(t, \mu_{5}, \gamma\right)=- & \delta_{5}\left(\mu_{5}, \gamma, E P O, \frac{d}{d t} E P O\right) P_{5}\left(t, \mu_{5}, \gamma\right)-\rho_{5} \frac{\partial}{\partial \mu_{5}} P_{5}\left(t, \mu_{5}, \gamma\right) \\
& -\frac{\partial}{\partial \gamma}\left[h_{5}\left(F e_{p l}, \gamma\right) P_{5}\left(t, \mu_{5}, \gamma\right)\right]
\end{aligned}
$$

with initial condition

$$
P_{5}\left(0, \mu_{5}, \gamma\right)=P_{5}^{\text {init }}\left(\mu_{5}, \gamma\right)
$$

and boundary conditions at $\left(\mu_{4}\right)_{f}=2, \mu_{5}=0$

$$
P_{5}(t, 0, \gamma)=P_{4}(t, 2, \gamma)
$$


and

$$
P_{5}\left(t, \mu_{5}, 0\right)= \begin{cases}P_{4}(t, 2,0), & \mu_{5}=0 \\ P_{5}^{b d y, \gamma}\left(t, \mu_{5}\right), & \mu_{5} \neq 0\end{cases}
$$

\subsection{Hemoglobinization}

Cells in classes $P_{3}, P_{4}$ and $P_{5}$ participate in iron uptake for the purposes of synthesizing hemoglobin. As noted earlier, we use $\gamma$ as a unitless structure variable that indicates the level of iron in a given cell. Moreover, recall that $0 \leq \gamma \leq 2$, where $\gamma_{\text {full }}=1$ represents the amount of iron the "typical" RBC contains in a healthy individual. Finally recall when a cell divides, we assume that each of the daughter cells receives half of the parent cell's iron endowment.

In order to determine the rate of iron moving from the iron compartment and entering RBCs, we compare the rate of iron required for cells to be hemoglobinized at the maximum rate with the rate of iron available in the blood plasma (i.e., iron in the blood plasma compartment). Then we can determine if there is sufficient iron available to hemoglobinize at the maximum rate or if hemoglobinization is occurring in an iron-restricted fashion.

We assume that when there is an abundance of iron available, cells can obtain a full complement of iron in three days [17]. Cells begin the hemoglobinization process in class $P_{3}$. Cells reside in this class for one day and they undergo cell division at a rate of 1 division per day. After cells transition out of class $P_{3}$, they no longer undergo cell division (i.e., cells in classes $P_{4}$ and $P_{5}$ do not divide). While we model hemoglobinization and proliferation as continuous processes, the following considerations in discrete time nodes indicate that the maximum rate of iron uptake should be set to $\frac{2}{5} \gamma_{\text {full }}$ days $^{-1}$ so that a cell could attain a full iron complement in three days' time.

$$
\begin{aligned}
\text { day 0: } & \gamma=0 \\
\text { day } 1 \text { before cell division: } & \gamma=\frac{2}{5} \gamma_{f u l l}=\frac{2}{5} \\
\text { day 1 after cell division: } & \gamma=\frac{1}{5} \gamma_{f u l l}=\frac{1}{5} \\
\text { day 2: } & \gamma=\frac{1}{5} \gamma_{f u l l}+\frac{2}{5} \gamma_{\text {full }}=\frac{3}{5} \\
\text { day 3: } & \gamma=\frac{3}{5} \gamma_{\text {full }}+\frac{2}{5} \gamma_{\text {full }}=1
\end{aligned}
$$

Cells in class $P_{4}$ can uptake iron at this rate until their iron state reaches $\gamma_{f u l l}$, at which point they stop taking in iron. Reticulocytes in class $P_{5}$ (i.e., cells in class $P_{5}$ with maturity level $0 \leq \mu_{5} \leq 2$ ) are circulating in the blood. While they are still capable of iron uptake until they reach iron state $\gamma_{f u l l}=1$, the rate of uptake is smaller [17], so we multiply the rate for class $P_{4}$ by the constant $0<k_{5}<1$. Therefore we define the maximum iron uptake rate 
functions $h_{3}^{\max }(\gamma), h_{4}^{\max }(\gamma)$, and $h_{5}^{\max }\left(\mu_{5}, \gamma\right)$, depicted in Figure 9, by

$$
\begin{aligned}
h_{3}^{\max }(\gamma) & =\frac{2}{5} \\
h_{4}^{\max }(\gamma) & =\left(\frac{2}{5}\right) \cdot \frac{\left(\gamma_{\text {full }}\right)^{k_{h, 4, \text { max }}}}{\left(\gamma_{\text {full }}\right)^{k_{h, 4, \text { max }}}+(\gamma)^{k_{h, 4, \text { max }}}}=\frac{2}{5} \cdot \frac{1}{1+\gamma^{k_{h, 4, \text { max }}}} \\
h_{5}^{\max }\left(\mu_{5}, \gamma\right) & = \begin{cases}k_{5} h_{4}^{\max }(\gamma), & 0 \leq \mu_{5} \leq 2 \\
0, & \mu_{5}>2 .\end{cases}
\end{aligned}
$$

Then the rate of iron needed if every cell becomes hemoglobinized at its maximum rate is

$$
\begin{aligned}
\rho_{\text {Fe,needed }}=\left(\max \text { rate iron needed in class } P_{3}\right)+\left(\max \text { rate iron needed in class } P_{4}\right) \\
+\left(\text { max rate iron needed in class } P_{5}\right) \\
=k_{F e}\left[\int_{0}^{\gamma_{f}} \int_{0}^{1} \frac{2}{5} P_{3}\left(t, \mu_{3}, \gamma\right) d \mu_{3} d \gamma\right. \\
+\int_{0}^{\gamma_{f}} \int_{0}^{2} h_{4}^{\max }(\gamma) P_{4}\left(t, \mu_{4}, \gamma\right) d \mu_{4} d \gamma \\
\left.\quad+\int_{0}^{\gamma_{f}} \int_{0}^{2} h_{5}^{\max }\left(\mu_{5}, \gamma\right) P_{5}\left(t, \mu_{5}, \gamma\right) d \mu_{5} d \gamma\right]
\end{aligned}
$$

where $k_{F e}$ is the amount of iron in one billion red blood cells with a full iron endowment.

We compare this with the rate of iron available for erythropoiesis at time $t$. We assume that the iron available for erythropoiesis is proportional to amount of iron in the blood plasma compartment. Hence, the rate of iron availability is given by

$$
\rho_{F e, a v a i l}=k_{F e, e f f} F e_{p l}(t),
$$

\section{Maximum Iron Uptake Rates for classes RBC}

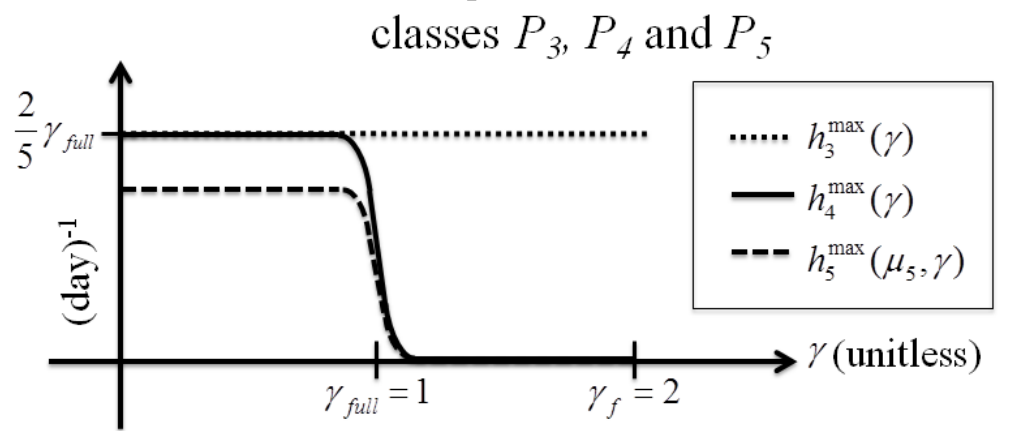

Figure 9: Maximum iron uptake rates where $0 \leq \mu_{5} \leq 2$. 
where $0<k_{F e, e f f} \leq 1$ days $^{-1}$.

We define the iron availability rate fraction, $0<k_{F e, \text { avail }} \leq 1$, to be

$$
k_{F e, \text { avail }}= \begin{cases}1, & \rho_{F e, \text { needed }} \leq \rho_{F e, \text { avail }}, \\ \frac{\rho_{F e, \text { avail }}}{\rho_{F e, \text { needed }},}, & \rho_{F e, \text { needed }}>\rho_{F e, \text { avail }} .\end{cases}
$$

Then the actual rates of iron uptake are given by

$$
h_{i}\left(F e_{p l}, \gamma\right)=k_{F e, \text { avail }} h_{i}^{\max }(\gamma), \quad i=3,4,5 .
$$

\subsection{EPO compartment}

While healthy individuals produce EPO in response to a decrease in blood oxygen, patients whose kidneys have only minimal function produce only a small basal level of EPO in the kidney and liver [21]. They receive rHuEPO intravenously during their dialysis treatments. In this model, we assume the use of a bioidentical rHuEPO; we will not distinguish between rHuEPO and endogenous EPO with respect to their action.

We assume the rate of endogenous EPO production in the liver and kidney to be constant. We will assume that EPO clearance is proportional to the amount present. Thus we have

$$
\frac{d}{d t} E P O(t)=\rho_{E P O, \text { endog }}+\rho_{E P O, \text { exog }}(t)-\frac{\ln 2}{t_{1 / 2}} E P O(t),
$$

with initial condition

$$
E P O(0)=E_{0},
$$

where $\rho_{E P O, \text { endog }}$ is the (assumed constant) rate of endogenous EPO production, $\rho_{E P O, \text { exog }}(t)$ is the rate of exogenous EPO provided during treatment, and $t_{1 / 2}$ is the half-life of EPO.

\section{$5 \quad$ Iron Model}

We now introduce the second portion of our model, the iron compartments. Iron is required to make hemoglobin, the protein that gives erythrocytes the ability to carry oxygen. It is also the protein that gives erythrocytes their characteristic red color. If iron is not available during erythropoiesis, the result is lighter-colored (hypochromic) erythrocytes with reduced capacity to carry oxygen.

Control of iron in the body is a strictly regulated process, in part because there is no pathway for the excretion of excess iron $[16,45]$. When red blood cells age, they become enveloped by macrophages in the spleen. The iron from their hemoglobin is then recaptured and sent to the bone marrow for use in making hemoglobin for new erythrocytes. This recycling process is very efficient and is the main source of iron to erythropoiesis [40]. In much smaller quantities, iron is absorbed from diet and is transferred in and out of storage in the liver. The only losses to the system are from sweating, cells being shed, blood losses, etc.

The major regulator of iron homeostasis is the hormone hepcidin, which is produced in the liver $[16,30,45]$. Hepcidin's role in iron homeostasis has only been recently studied; the 


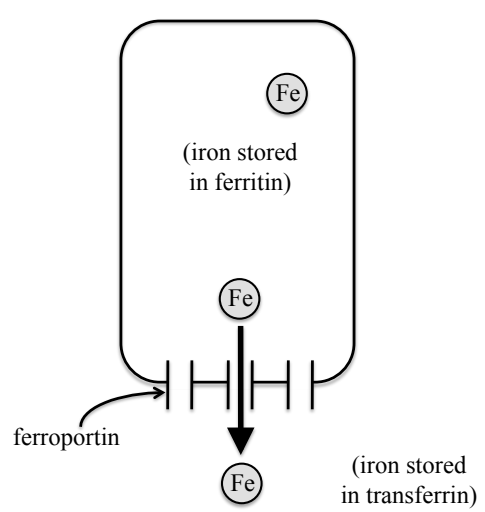

(a) Ferroportin is required for the transport of iron out of cells.

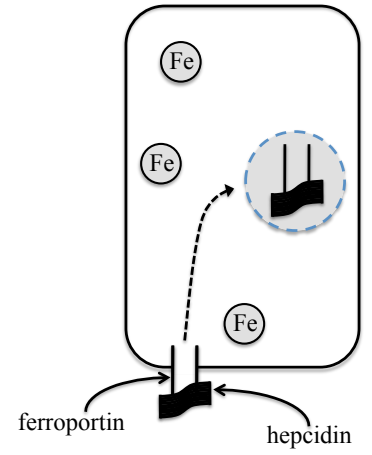

(b) Hepcidin is the major regulator of iron transport out of cells.

Figure 10: Iron regulation at a cellular level.

mechanisms and pathways leading to the production and action of hepcidin are only beginning to be understood.

The protein ferroportin is required to transport iron out of a cell and into the plasma. Hepcidin binds to ferroportin and causes the complex to be absorbed into the cell, effectively interrupting the transport of iron into the blood plasma, as depicted in Figure 10. Hence, an increase in hepcidin level causes iron to remain in cells and not be released into the blood plasma (where it could be used in RBC production).

Hepcidin production is increased in response to high iron level in the blood plasma and/or liver and is decreased in response to erythropoietic activity [16, 30]. Production is also increased in the presence of certain cytokines which are released due to inflammation in the body $[16,45]$. It is thought that this might be a defense mechanism against foreign organisms which may need iron to reproduce. Since patients in ESRD commonly have other health problems, they often have higher than normal levels of inflammation. Thus, they may produce higher than normal levels of hepcidin and as a result, even if there is enough iron in the body, it may not be available for erythropoiesis because it cannot leave the cells and enter the plasma [1].

We propose a model of ordinary differential equations for the iron model using the conceptual model given in Figure 11.

\subsection{Hepcidin}

We choose $H(t)$ to represent plasma hepcidin level at time $t$, with

$$
0 \leq H(t) \leq 1
$$

A hepcidin level of 0 indicates little or no hepcidin present. In this situation, iron can pass unencumbered across a cell membrane and enter the iron in the blood plasma compartment. 


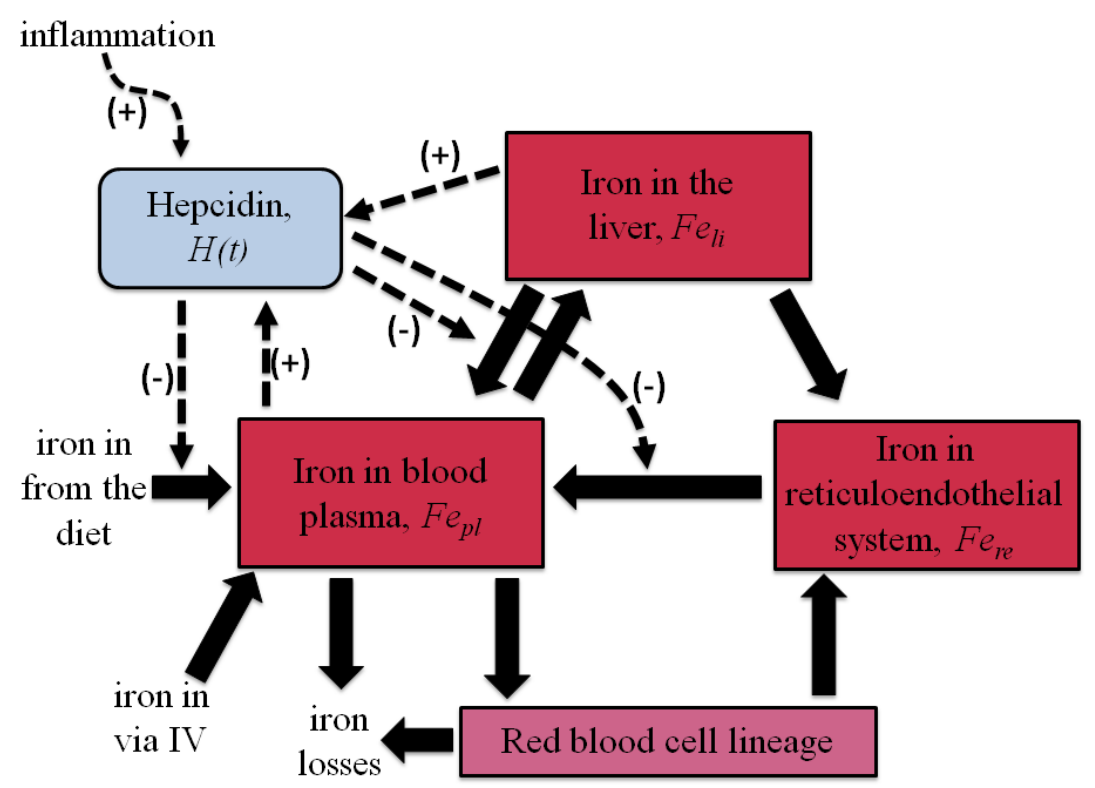

Figure 11: Iron Model. Note that the states variables are $H, F e_{l i}, F e_{p l}$ and $F e_{r e}$.

A hepcidin level of 1 indicates a maximum hepcidin level; when hepcidin is at its maximum level, iron cannot cross a cell membrane to enter the blood plasma. In order to ensure that $0 \leq H \leq 1$, we use characteristic functions that are "turned on" when a condition is true and "off" when it is false. For example,

$$
\chi_{\{H<1\}}(t)=\chi_{\{\tau \mid H(\tau)<1\}}(t)= \begin{cases}1, & H(t)<1 \\ 0, & H(t) \geq 1\end{cases}
$$

We assume the following:

1. Hepcidin is produced in response to high levels of iron; how this is measured by the body is unclear. We assume that production is increased in response to high levels in both the liver and in the plasma. The relative size of these two responses can be adjusted by way of the coefficients $k_{F e, p l}$ and $k_{F e, l i}$ :

rate of increase in hepcidin due to high level of iron in plasma $=k_{F e, p l} F e_{p l}(t) \chi_{\{H<1\}}$,

rate of increase in hepcidin due to high level of iron in liver $=k_{F e, l i} F e_{l i}(t) \chi_{\{H<1\}}$,

with $0 \leq k_{F e, p l}, k_{F e, l i} \leq 1$. The characteristic functions in these terms allow hepcidin level to increase to $H=1$ in response to high level of iron in the plasma or liver, but do not allow hepcidin level to increase above $H=1$.

2. Hepcidin production is increased in the presence of inflammation, but the specific pathways that cause this response are still under investigation. We assume that the input $I(t)$ is a measure of the overall inflammation state of the body (a broad simplification that should be revisited in future model iterations) such that $0 \leq I \leq 1$. Therefore,

rate of increase in hepcidin due to inflammation $=k_{\text {infl }} I(t) \chi_{\{H<1\}}$, 
where $0 \leq k_{\text {infl }} \leq 1$

3. While hepcidin is actually "consumed" as it is used to prevent the action of ferroportin, we initially assume a clearance proportional the amount present.

Hence, we model hepcidin level by

$\frac{d H}{d t}(t)=k_{i n f l} I(t) \chi_{\{H<1\}}(t)+k_{F e, p l} F e_{p l}(t) \chi_{\{H<1\}}(t)+k_{F e, l i} F e_{l i}(t) \chi_{\{H<1\}}(t)-k_{H} H(t) \chi_{\{H>0\}}(t)$,

where $0 \leq k_{H} \leq 1$

\subsection{Iron in the reticuloendothelial system}

The reticuloendothelial system consists of macrophages in the spleen and other cells that collect/envelop old and damaged cells and reclaim their iron. This includes any cell in the red blood cell lineage that dies, as well as liver cells that become senescent and/or damaged.

Based on our model for RBC dynamics, the rate of iron in to the reticuloendothelial system from RBC lineage is

$$
\begin{gathered}
k_{F e}\left[\int_{0}^{\gamma_{f}} \int_{0}^{1} \delta_{3}\left(\mu_{3}, E P O\right) \gamma P_{3}\left(t, \mu_{3}, \gamma\right) d \mu_{3} d \gamma\right. \\
+\int_{0}^{\gamma_{f}} \int_{0}^{2} \delta_{4}\left(\mu_{4}, E P O\right) \gamma P_{4}\left(t, \mu_{4}, \gamma\right) d \mu_{4} d \gamma \\
+\int_{0}^{\gamma_{f}} \int_{0}^{100}\left[\delta_{5, \gamma}\left(\mu_{5}, \gamma\right)+\delta_{5, \mu}\left(\mu_{5}\right)\right. \\
\left.\left.+\delta_{5, \text { neo }}\left(\mu_{5}, E P O, \frac{d}{d t} E P O\right)\right] \gamma P_{5}\left(t, \mu_{5}, \gamma\right) d \mu_{5} d \gamma\right]
\end{gathered}
$$

where $k_{F e}$ is the amount of iron in one billion RBCs with a "normal" iron endowment. Notice that for class $P_{5}$ we do not recycle iron from deaths due to blood losses.

The liver serves as a storage area for iron. Iron residing in liver cells that die is recaptured and ends up in the reticuloendothelial system. Hence, we assume that

rate of iron in to the reticuloendothelial system from the liver $=k_{l i \rightarrow r e} F_{l i}(t)$,

where $0 \leq k_{l i \rightarrow r e} \leq 1$.

Reticuloendothelial cells require ferroportin to transfer iron from within the cell into the blood plasma. Hepcidin negatively regulates this transport; when hepcidin level is high, the amount of iron that can leave liver cells and enter the blood plasma is low. We assume that

rate of iron out of the reticuloendothelial system in to the blood plasma $=k_{r e \rightarrow p l}(1-H(t)) F e_{r e}(t)$, where $0 \leq k_{r e \rightarrow p l} \leq 1$ 
Thus, we have

$$
\begin{aligned}
\frac{d F e_{r e}}{d t}=k_{F e} & {\left[\int_{0}^{\gamma_{f}} \int_{0}^{1} \delta_{3}\left(\mu_{3}, E P O\right) \gamma P_{3}\left(t, \mu_{3}, \gamma\right) d \mu_{3} d \gamma\right.} \\
& +\int_{0}^{\gamma_{f}} \int_{0}^{2} \delta_{4}\left(\mu_{4}, E P O\right) \gamma P_{4}\left(t, \mu_{4}, \gamma\right) d \mu_{4} d \gamma \\
& \left.+\int_{0}^{\gamma_{f}} \int_{0}^{100}\left[\delta_{5, \gamma}\left(\mu_{5}, \gamma\right)+\delta_{5, \mu}\left(\mu_{5}\right)+\delta_{5, \text { neo }}\left(\mu_{5}, E P O, \frac{d}{d t} E P O\right)\right] \gamma P_{5}\left(t, \mu_{5}, \gamma\right) d \mu_{5} d \gamma\right] \\
& +k_{l i \rightarrow r e} F e_{l i}-k_{r e \rightarrow p l}(1-H) F e_{r e} .
\end{aligned}
$$

\subsection{Iron in the Liver}

Iron enters the liver (for storage) from the blood plasma when the level of iron in the blood plasma is high and leaves storage when the level of iron in the blood plasma is low. We model these rates with sigmoid functions as follows:

rate of iron into liver from the blood plasma compartment $=K_{p l \rightarrow l i}\left(F e_{p l}(t)\right) F e_{p l}(t)$,

where

$$
K_{p l \rightarrow l i}\left(F e_{p l}\right)=k_{\max }\left(1-\frac{\left(c_{p l \rightarrow l i}\right)^{k_{p l \rightarrow l i}}}{\left(c_{p l \rightarrow l i}\right)^{k_{p l \rightarrow l i}}+\left(F e_{p l}\right)^{k_{p l \rightarrow l i}}}\right)
$$

and

rate of iron out of the liver into the blood plasma

$$
=K_{l i \rightarrow p l}\left(F e_{p l}(t)\right)(1-H(t)) F e_{l i}(t)
$$

where

$$
K_{l i \rightarrow p l}\left(F e_{p l}\right)=k_{\max }\left(\frac{\left(c_{l i \rightarrow p l}\right)^{k_{l i \rightarrow p l}}}{\left(c_{l i \rightarrow p l}\right)^{k_{l i \rightarrow p l}}+\left(F e_{p l}\right)^{k_{l i \rightarrow p l}}}\right) .
$$

Notice that $1-H$ appears in the expression for rate of iron into the blood plasma compartment from the liver compartment because hepcidin inhibits the action of ferroportin in allowing the transport of iron out of the liver. The functions $k_{p l \rightarrow l i}\left(F e_{p l}\right)$ and $k_{l i \rightarrow p l}\left(F e_{p l}\right)$ are depicted in Figure 12.

When cells in the liver die, the iron in those cells is collected by cells in the reticuloendothelial system. We model this as

rate of iron out of the liver in to the reticuloendothelial system $=k_{l i \rightarrow r e} F e_{l i}(t)$.

Hence, the time rate of change of iron in the liver is given by

$$
\frac{d F e_{l i}}{d t}=K_{p l \rightarrow l i}\left(F e_{p l}(t)\right) F e_{p l}(t)-K_{l i \rightarrow p l}\left(F e_{p l}(t)\right)(1-H(t)) F e_{l i}(t)-k_{l i \rightarrow r e} F e_{l i}(t) .
$$




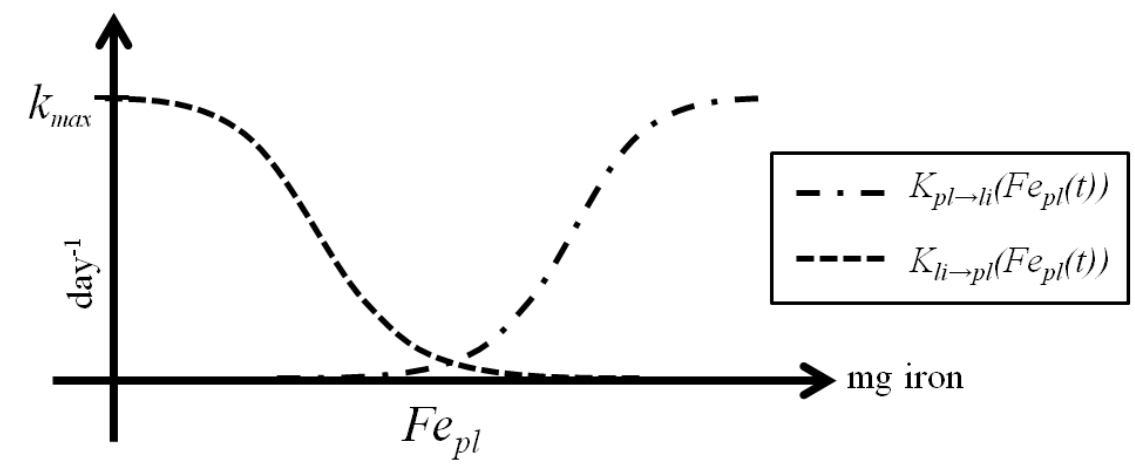

Figure 12: Rate coefficients for iron in and out of storage (i.e., iron into the liver from the blood plasma and iron into the blood plasma from the liver).

\subsection{Iron in the Blood Plasma}

Iron in the blood plasma is the main source of iron for erythropoiesis. Iron added to blood plasma during intravenous treatment is denoted $F e_{e x}(t)$. Iron also enters the blood plasma from the reticuloendothelial system, the liver, and the intestines from diet. Since each of these requires ferroportin to transfer the iron from within the cell to the plasma, the associated rates of transfer are all subject to the negative regulatory effect of hepcidin.

Iron enters the intestines from a patient's dietary intake. We define

$$
\rho_{\text {iron }, \text { diet }}=\text { maximum rate of iron coming from diet. } \mathrm{mg} / \text { day }
$$

Iron in intestinal cells must be transported into the blood plasma via ferroportin. Hence, the rate of iron into the blood plasma is limited by the hepcidin level. Thus, we assume

$$
\text { rate of iron into blood plasma from diet }=k_{\text {diet }}(1-H) \rho_{\text {iron,diet }} \text {, }
$$

where $0 \leq k_{\text {diet }} \leq 1$.

We define

$$
F e_{e x}(t)=\text { rate of iron into plasma from intravenous treatment }
$$

and

$$
\rho_{F e, p l, l o s s}(t)=\text { rate of iron lost via blood plasma losses. }
$$

The rates of iron (i) into blood plasma from the reticuloendothelial system, (ii) into the blood plasma from the liver, (iii) into the liver from the blood plasma, and (iv) into the red blood cell lineage were all described previously. Thus, we have the following equation for iron 
in the blood plasma:

$$
\begin{aligned}
& \frac{d F e_{p l}}{d t}=\text { rate of iron into plasma from diet } \\
& + \text { rate of iron into plasma from reticuloendothelial system } \\
& + \text { rate of iron into plasma from liver } \\
& + \text { rate of iron into plasma from intravenous treatment } \\
& \quad \text { rate of iron into liver from plasma } \\
& \quad \text { - rate of iron into RBC lineage from plasma } \\
& \quad-\text { rate of iron lost via blood plasma losses } \\
& =k_{d i e t}(1-H) \rho_{i r o n, d i e t} \\
& +k_{r e \rightarrow p l}(1-H) F e_{r e}(t) \\
& +K_{l i \rightarrow p l}(1-H) F e_{l i}(t) \\
& +F e_{e x}(t) \\
& -K_{p l \rightarrow l i}\left(F e_{p l}\right) F e_{p l}(t) \\
& -k_{F e}\left[h_{3}\left(F e_{p l}, \gamma\right)+h_{4}\left(F e_{p l}, \gamma\right)+h_{5}\left(F e_{p l}, \gamma\right)\right] \\
& \quad-\rho_{F e, p l, l o s s}(t) .
\end{aligned}
$$

\section{Summary}

We have developed a model for both red blood cell and iron dynamics in patients in ESRD undergoing hemodialysis. In particular, our model consists of the RBC precursor and circulating fragmentation equations (5)-(9) with appropriate initial and boundary conditions, coupled with (10)-(14). We use structure variables to account for the age and iron endowment of cells in the red blood cell lineage in the fragmentation equations for $P_{1}$ through $P_{5}$. The model also permits investigation of the effects of neocytolysis and inflammation on red blood cell dynamics. The coupled fragmentation and EPO, iron, hepcidin with inflammation in-

put are nontrivial to solve. We are currently carrying out simulations on both the continuous model presented here, and a corresponding discrete time version, in verification and validation studies.

\section{Acknowledgments:}

This research was supported in part by the National Institute of Allergy and Infectious Disease under grant NIAID 9R01AI071915. The authors would like to thank Dustin Kapraun, Peter Kotanko, Franz Kappel and Doris Furtinger for stimulating and fruitful conversations during the course of their efforts. 


\section{References}

[1] Plasma hepcidin levels are elevated but responsive to erythropoietin therapy in renal disease, Kidney International, 75 (2009), 976-981.

[2] A. Ackleh, K. Deng, K. Ito, and J. Thibodeaux, A structured erythropoiesis model with nonlinear cell maturation velocity and hormone decay rate, Mathematical Biosciences, 204 (206), 21-48.

[3] Clarence P. Alfrey and Stephen Fishbane, Implications of neocytolysis for optimal management of anemia in chronic kidney disease, Nephron Clinical Practice, 106 (2007), c149-c156.

[4] Clarence P. Alfrey, Mark M. Udden, Carolyn Leach-Huntoon, Theda Driscoll, and Mark H. Pickett, Control of red blood cell mass in spaceflight, Journal of Applied Physiology, 81 (1996), 98-104.

[5] H. T. Banks, Karen M. Bliss, Peter Kotanko, and Hien Tran, A computational model of red blood cell dynamics in patients with chronic kidney disease, CRSC Tech Report TR11-03, N.C. State University, Raleigh, NC, February, 2011.

[6] H. T. Banks, C. Cole, P. Schlosser, and H. T. Tran, Modeling and optimal regulation of erythropoiesis subject to benzene intoxication, Mathematical Biosciences and Engineering, 1 (2004), 15-48.

[7] J. Belair, M. Mackey, and J. Mahaffy, Age-structured and two-delay models for erythropoiesis, Mathematical Biosciences, 128 (1995), 317-346.

[8] Karen M. Bliss. Modeling of Red Blood Cell Dynamics in Patients with Chronic Kidney Disease, Ph D Thesisi, N. C. State University, Raleigh, NC, May, 2011.

[9] Francesco M. Van Bockxmeer and Evan H. Morgan, Transferrin receptors during rabbit reticulocyte maturation, Biochemica et Biophysica Acta, 584 (1979), 76-83.

[10] Robert T. Card and Lewis R. Weintraub, Metabolic abnormalities of erythrocytes in severe iron deficiency, Blood, 37 (1971), 725-732.

[11] Chung-Che Chang, Yayan Chen, Kapil Modi, Omar Awar, Clarence P. Alfrey, and Lawrence Rice, Changes of red blood cell surface markers in a blood doping model of neocytolysis, Journal of Investigative Medicine, 57 (2009), 650-654.

[12] Stefan N. Constantinescu, Mechanism of erythropoietin receptor activation, In S. G. Elliot, M. A. Foote, and G. Molineaux, editors, Erythropoietins, Erythropoietic Factors and Erythropoiesis, Birkhauser Verlag/Switzerland, 2009, pp. 175-196.

[13] Maria Diez-Ewald and Miguel Layrisse, Mechanisms of hemolysis in iron deficiency anemia: further studies, Blood, 32 (1968), 884-894. 
[14] Z. Farid, J. H. Nichols, S. Bassily, and A. R. Schulert, Blood loss in pure Ancylostoma Duodenale infection in egyptian farmers, American Journal of Tropical Medicine and Hygiene, 14 (1965), 375-378.

[15] Janet L. Frazier, Jennifer H. Caskey, Mark Yoffe, and Paul A. Seligman, Studies of the transferrin receptor on both human reticulocytes and nucleated human cells in culture, Journal of Clinical Investigation, 69 (1982), 853-865.

[16] Tomas Ganz, Iron homeostasis: Fitting the puzzle pieces together, Cell Metabolism, 7 (2008), 288-290.

[17] John P. Greer, John Foerster, George M. Rodgers, Frixos Paraskevas, Bertil Glader, Daniel A. Arber, and Jr. Robert T. Means, Wintrobe's Clinical Hematology, Lippincott Williams and Wilkins, 12th edition, 2009.

[18] Connie J. Gregory and Allen C. Eaves, Human marrow cells capable of erythropoietic differentiation in vitro: definition of three erythroid colony responses, Blood, 49 (1977), 855-864.

[19] Connie J. Gregory and Allen C. Eaves, Three stages of erythropoietic progenitor cell differentiation distinguished by a number of physical and biologic properties, Blood, $\mathbf{5 1}$ (1978), 527-537.

[20] L. Israels and E. Israels, Erythropoiesis: an overview, In Erythropoietins and Erythropoiesis: Molecular, Cellular, Preclinical and Clinical Biology, Birkhäuser Verlag, 2003.

[21] W. Jelkmann, Erythropoietin: structure, control of production, and function, Physiol Rev., 72 (1992), 449-489.

[22] Mark J. Koury and Prem Ponka, New insights in erythropoiesis: The roles of Folate, Vitamin B12, and Iron, Annual Review of Nutrition, 24 (2004), 105-131.

[23] Sanford B. Krantz, Erythropoietin, Blood, 77 (1991), 419-434.

[24] Joseph Ly, Rosa Marticorena, and Sandra Donnelly, Red blood cell survival in chronic renal failure, American Journal of Kidney Diseases, 44 (2004), 715-719.

[25] I. Macdougall, Use of recombinant erythropoietins in the setting of renal disease, In S.G. Elliot G. Molineaux, M.A. Foote, eds., Erythropoietins and Erythropoiesis: Molecular, Cellular, Preclinical and Clinical Biology, Birkhäuser Verlag, 2003, pp. 153-159.

[26] I. Macdougall and A. Cooper, Erythropoietin resistance: the role of inflammation and pro-inflammatory cytokines, Nephrol. Dial. Transplant., 17 (2002), 39-43.

[27] J. Mahaffy, J. Belair, and M. Mackey, Hematopoietic model with moving boundary condition and state dependent delay: applications in erythropoiesis, Journal of Theoretical Biology, 190 (1998), 135-146.

[28] Cesar F. Merino, Studies on blood formation and destruction in the polycythemia of high altitude, Blood, 5 (1950), 1-31. 
[29] National Kidney Foundation Kidney Disease Outcomes Quality Initiative, KDOQI Clinical Practice Guidline and Clinical Practice Recommendations for Anemia in Chronic Kidney Disease: 2007 Update of Hemoglobin Target, American Journal of Kidney Diseases, 50 (2007), 471-530.

[30] Elizabeta Nemeth and Tomas Ganz, The role of hepcidin in iron metabolism, Acta Haematologica, 122 (2009), 78-86.

[31] R. Ramakrishnan, W. Cheung, M. Wacholtz, N. Minton, and W. Jusko, Pharmacokinetic and pharmacodynamic modeling of human erythropoietin after single and multiple doses in healthy volunteers, The Journal of Clinical Pharmacology, 44 (2004), 991-1002.

[32] Lawrence Rice and Clarence Alfrey, The negative regulation of red cell mass by neocytolysis: Physiologic and pathophysiologic manifestations, Cellular Physiology and Biochemistry, 15 (2005), 245-250.

[33] Lawrence Rice, Clarence P. Alfrey, Theda Driscoll, Carl E. Whitley, David L. Hachey, and Wadi Suki, Neocytolysis contributes to the anemia of renal disease, American Journal of Kidney Disease, 33 (1999), 59-62.

[34] Lawrence Rice, Wilson Rulz, Theda Driscoll, Carl E. Whitley, Rosario Tapia, David L. Hachey, Gustavo F. Gonzales, and Clarence P. Alfrey, Neocytolysis on descent from altitude: A newly recognized mechanism for the control of red cell mass, Annals of Internal Medicine, 134 (2001), 652-656.

[35] Bernadette F. Rodak, George A. Fritsma, and Kathryn Doig], Hematology: Clinical Principles and Applications, Saunders Elsevier, 3rd ed, 2007.

[36] Pradeep Sathyanarayana, Arvind Dev, Jing Fang, Estelle Houde, Olga Bogacheva, Oleg Bogachev, Madhu Menon, Sarah Browne, Anamika Pradeep, Christine Emerson, and Don M. Wojchowski, Epo receptor circuits for primary erythroblast survival, Blood, 111 (2008), 5390-5399.

[37] K. Sawada, S. B. Krantz, S-H. Dai, S. T. Koury, S. T. Horn, A. D. Glick, and C. I. Civin, Purification of human blood burst-forming units-erythroid and demonstration of the evolution of erythropoietin receptors, Journal of Cellular Physiology, 142 (1990), 219-230.

[38] K. Sawada, S. B. Krantz, J. S. Kans, E. N. Dessypris, S. Sawyer, A. D. Glick, and C. I. Civin, Purification of human erythroid colony-forming units and demonstration of specific binding of erythropoietin, Journal of Clinical Investigation, 80 (1987), 357-366.

[39] A. B. Shaw, Haemolysis in chronic renal failure, British Medical Journal, 2 (1967), $213-216$.

[40] A. Simmons], Basic Hematology, Charles C Thomas, Springfield, Illinois, 1973.

[41] J. H. Stewart, Haemolytic anemia in acute and chronic renal failure, QJM, 36 (1967), $85-105$. 
[42] U.S. Renal Data System, USRDS 2008 Annual Data Report: Atlas of Chronic Kidney Disease and End-Stage Renal Disease in the United States, National Institutes of Health, National Institute of Diabetes and Digestive and Kidney Diseases, Bethesda, MD, 2008.

[43] M. M. Udden, T. B. Driscoll, M. H. Pickett, C. S. Leach-Huntoon, and C. P. Alfrey, Decreased production of red blood cells in human subjects exposed to microgravity, Journal of Laboratory and Clinical Medicine, 125 (1995), 442-449.

[44] M. C. Verloop, M. Van der Wolk, and A. J. Heier, Radioactive iron studies in patients with iron deficiency anemia with concurrent abnormal hemolysis, Blood, 15 (1960), 791806.

[45] Mark Westerman, Gordana Olbina, Vaughn Ostland, Elizabeta Nemeth, and Tomas Ganz, Hepcidin: An emerging biomarker for iron disorders, inflammatory diseases, and infections, Proceedings of SPIE, 7666 (2010), 766617-1.

[46] S. Woo, W. Krzyzanski, and W. Jusko, Pharmacokinetic and pharmacodynamic modeling of recombinant human erythropoietin after intravenous and subcutaneous administration in rats, Journal of Pharmacology and Experimental Therapeutics, 319 (2006), 1297-1306.

[47] Hong Wu, Xin Liu, Rudolf Jaenisch, and Harvey F. Lodish, Generation of committed erythroid bfu-e and cfu-e progenitors does not require erythropoietin or the erythropoietin receptor, Cell, 83 (1995), 59-67. 